\title{
IL CIBO DEGLI ALTRI. VIAGGIATORI MEDIEVALI ALLA TAVOLA DEGLI STRANIERI
}

\author{
PAOLO CHIESA (*)
}

SunTO. - Il cibo può diventare mezzo di confronto fra popoli e culture: il bisogno di mangiare, che è comune a tutti gli uomini, si realizza in alimenti e consuetudini diverse, e su tali differenze si misurano vicinanze e distanze. Questo assunto è esemplificato nelle esperienze di alcuni viaggiatori medievali, che vengono a contatto con consuetudini alimentari molto diverse dalle loro, e attraverso di esse conoscono e rappresentano altre civiltà. I casi citati sono quelli di Guglielmo di Rubruk, viaggiatore in Mongolia nel XIII sec., che si adatta al cibo dei popoli che incontra non disdegnandone la qualità; di Liutprando di Cremona, ambasciatore a Costantinopoli nel X sec., per il quale al contrario le usanze alimentari bizantine sono prova del degrado morale e culturale di quella società; e di Marco Polo (e Giovanni di Marignolli), viaggiatori in Cina nel XIV sec., per i quali il cibo esotico vien visto con le attenzioni del mercante, ma diventa presto uno strumento per stupire il lettore.

$$
* * *
$$

ABSTRACT. - Food can be a means of comparison between peoples and cultures. Everyone needs to eat, but every people has its own foods and alimentary customs, different from others; realizing these differences is a way for recognizing or highlighting affinities or distances. This paper exemplifies the assumption, reporting some experiences of medieval travellers, who came into contact with exotic food habits, very far from European customs. William of Rubruk, traveller in Mongolia in the thirteenth century, describes the food of the peoples he meets without disdaining its quality; on the contrary, for Liutprand of Cremona, ambassador to Constantinople in the tenth century, Byzantine food customs are evidence of the cultural and moral degradation of that society; Marco Polo and John of Marignolli, travellers to China in the thirteenth and fourteenth centuries, evaluated exotic food which merchant's eyes, but also with the aim to impress the reader on their extraordinary journeys.

(*) Università degli Studi di Milano, Italia. E-mail: paolo.chiesa@unimi.it 
È esperienza comune di ciascuno di noi che nel racconto di un viaggio si dia ampio spazio a riferire le abitudini alimentari dei luoghi visitati. Personalmente non ricordo nessuna esperienza di viaggio, mia o altrui, in cui non siano state fatte osservazioni sulla cucina del posto, nelle sue caratteristiche e nella sua qualità; così come è impossibile trovare una guida turistica che non presenti una sezione sul cibo del territorio trattato, nel bene e nel male (cosa mangiare, cosa evitare di mangiare, cosa mangiare con certe precauzioni ecc.). Il nutrirsi è un'attività primaria e indispensabile dell'uomo, e parlare dell'alimentazione è dunque importante; ma quando l'alimentazione è associata al viaggio - cioè all'uscire dal mondo cui siamo abituati e all'entrare in un contesto diverso e poco familiare - essa diventa un potente strumento di confronto culturale e di identificazione antropologica. Tutti gli uomini hanno l'obbligo di mangiare; questa necessità è dunque un vincolo ineludibile, un vincolo egualitario, e perciò anche una chiave universale attraverso la quale misurare affinità e distanze. Le variazioni che si registrano nel cibo, quelle che distinguono un popolo dall'altro, diventano un mezzo di riconoscimento: 1' "altro" si svela ai nostri occhi in quanto si nutre di cose diverse da quelle di cui ci nutriamo noi, o delle stesse cose ma preparate in un modo diverso, o con usanze e rituali diversi; tali differenze, per converso, permettono a chi viaggia di identificarsi nella confortante 'normalità' degli usi alimentari cui è avvezzo (alzi la mano chi, tornando da paesi anche piuttosto vicini, non ha detto: «finalmente un'insalata condita con l'olio d'oliva!», o «come mi mancavano gli spaghetti!», o «ecco un caffè come si deve!»).

Questo, ripeto, è esperienza di ognuno di noi, e universale nel nostro mondo. Ma l'universalità di questa esperienza non si limita ai nostri giorni: in ogni epoca della storia i viaggiatori sono stati prodighi di notizie sulle abitudini alimentari dei popoli "altri" che incontravano. Farò qualche esempio di viaggiatori medievali, sottolineando come il cibo diventi, nei loro racconti, non solo il risultato di una scoperta, ma anche uno strumento di indagine e di misura, e per converso un mezzo di identificazione; ma anche come essi siano in grado - grazie al confronto con il diverso - di riflettere sulle funzioni sociali del cibo, una consapevolezza indotta dalla situazione insolita in cui si erano venuti a trovare.

Nel 1253 il francescano fiammingo Guglielmo di Rubruk entra con quattro compagni nei territori dei Mongoli, che controllavano all'epoca un grande impero, forse il più grande mai esistito sulla terra. Lo scopo della spedizione è insieme missionario e diplomatico; il mandan- 
te e finanziatore è il re di Francia Luigi IX. Del suo viaggio Guglielmo ha lasciato una relazione personale e appassionata, probabilmente il più bel documento letterario di questo genere nell'intero medioevo ${ }^{1}$. Guglielmo parte dalle coste della Crimea e si dirige verso l'interno, attraverso la steppa; supera un rudimentale punto di confine costituito da presidio militare mongolo, e da questo momento lascia alle spalle il suo mondo e la sua civiltà occidentale, che rivedrà solo due anni dopo. «Il terzo giorno - così Guglielmo descrive la sua prima impressione incontrammo i tartari; quando arrivai fra loro mi sembrò davvero di entrare in un altro mondo» ${ }^{2}$. Qui egli assaggia per la prima volta la bevanda nazionale dei Mongoli, il qumiz, una sorta di yogurt prodotto con latte di cavalla lasciato fermentare e leggermente alcolico, che all' ${ }^{i}$ nizio gli fa uno strano effetto:

Quella sera l'uomo che ci faceva da guida ci diede da bere del qumiz; al primo sorso mi misi tutto a sudare per il disgusto e la sorpresa, perché non ne avevo mai bevuto. Tuttavia mi sembrò che avesse un buon sapore, ed effettivamente lo ha. ${ }^{3}$

A questa bevanda Guglielmo a poco a poco si affezionerà. Un anno e mezzo più tardi, quando sulla via del ritorno egli attraverserà l'ultimo accampamento mongolo, nelle valli del Caucaso, prima di entrare nelle più familiari terre degli Armeni e dei Turchi, si vedrà offrire dal capo locale, di nome Baiju (Baachu), una coppa di vino; Guglielmo dice di averlo accettato, naturalmente, e che era anche buono, ma rimpiange che non gli fosse stato offerto del qumiz, che afferma - «fa molto meglio a un uomo affamato $»^{4}$. Ciò che all'inizio era inusitato è diventato abitudine, il raccapriccio si è trasformato in apprezzamento; un'evoluzione che è anche il segno di un'accettazione

\footnotetext{
1 Citeremo l'opera dalla nostra edizione critica con traduzione italiana: Chiesa 2010.

2 «Tertia die invenimus Tartaros; inter quos cum intravi, visum fuit michi recto quod ingrederer quoddam aliud seculum» (Itiner. I 14, una frase ripresa poi a IX 1).

3 «Illo sero dedit nobis garcio qui ducebat nos bibere comos; ad cuius haustum totus sudavi propter horrorem et novitatem, quia numquam biberam de eo. Valde tamen sapidum videbatur michi, sicut vere est» (Itiner. IX 4).

4 «Fui in domo ipsius Baachu, et dedit nobis bibere vinum; ipse autem bibebat comos, quod ego etiam libencius bibissem, si dedisset michi: tamen vinum erat novum precipuum, sed comos plus satis proficit homini famelico» (Itiner. XXXVII 23).
} 
più profonda di un mondo e di uno stile di vita diversi, che Guglielmo da buon occidentale continua a considerare primitivi e barbarici, ma di cui sa cogliere la coerenza e i lati positivi.

Il qumiz, scoprirà presto Guglielmo, per i Mongoli non è solo una semplice bevanda, ma l'alimento fondamentale. Niente verdure, niente frutta, pochi cereali; moltissimo latte e moltissimi latticini, in grande varietà di preparazioni. Fra queste, il qumiz è la più apprezzata e diffusa, e Guglielmo lo descrive accuratamente ${ }^{5}$; ma ve ne sono anche altre, che il nostro viaggiatore presenta con la curiosità e l'interesse di chi le ha provate e ci tiene a farlo sapere:

Quanto al latte di vacca, prima di tutto ne estraggono il burro, e lo mettono a cuocere fino a che la parte liquida non è completamente evaporata; poi lo chiudono in budelli di montone predisposti allo scopo. Il burro, nonostante non venga salato, non irrancidisce per via della lunga cottura cui è stato sottoposto, e lo tengono da parte per l'inverno. Quanto rimane del latte dopo l'estrazione del burro lo fanno diventare il più acido possibile, poi lo fanno bollire fino a rapprendersi; poi lo seccano al sole finché diventa duro come un pezzo di ferro, e lo conservano in sacchi per l'inverno. D'inverno poi, quando manca il latte, pongono quel grumo acido, che chiamano grut, in un otre, vi versano sopra acqua calda e agitano il tutto energicamente finché il grumo non si scioglie; l'acqua assume così un sapore acido, e viene bevuta come surrogato del latte. Evitano invece in ogni modo di bere acqua. ${ }^{6}$

Di carne se ne consuma parecchia. La più pregiata è quella di montone, sempre e solo lessato; ma mangiano anche topi, ghiri e altra selvaggina, mentre gli schiavi devono accontentarsi del brodo di cot-

5 Itiner. IV 1-4.

6 «De lacte vaccino primo extrahunt butirum, et bulliunt illud usque ad perfectam decoctionem, et postea recondunt illud in ventribus arietinis quos ad hoc reservant. Et non ponunt sal in butiro, tamen propter magnam decoctionem non putrefit; et reservant illud contra hyemem. Residuum lac quod remanet post butirum permittunt acescere quantum acrius fieri potest et bulliunt illud, et coagulatur bulliendo; et coagulum illud siccant ad solem, et efficitur durum sicut scoria ferri; quod recondunt in saccis contra hyemem. Tempore hyemali, quando defficit eis lac, ponunt illud acrum coagulum, quod ipsi vocant grut, in utre, et superinfundunt aquam calidam, et concutiunt fortiter donec illud resolvatur in aqua, que ex illo efficitur tota acetosa; et illam aquam bibunt loco lactis. Summe cavent ne bibant aquam puram» (Itiner. IV 6). 
tura $^{7}$. Da questo montone lesso, che gli viene propinato spesso, Guglielmo cerca talvolta di astenersi, quando mangiare carne sarebbe una violazione di precetti religiosi, ma non sempre può farlo, perché non c'è altro. Il brodo gli sembra un buon compromesso:

La sera ci davano della carne, una spalla e delle coste di montone, e una razione di brodo da bere. Quando avevamo quel brodo di carne in abbondanza, ci saziavamo perfettamente, e mi pareva una bevanda quanto mai sana e nutriente. Il venerdì rimanevo digiuno fino a notte, senza prendere niente; poi ero costretto, con tristezza e dolore, a mangiare della carne. ${ }^{8}$

Pesce non se ne trova, perché non sanno come cucinarlo: solo i Mongoli più occidentali mangiano talvolta i grandi pesci che vivono nel Volga e nel Don (ma li fanno pescare ai Russi del posto), perché si possono buttare in una pentola d'acqua e lessare come si fa con i montoni'. Ma soprattutto hanno una quantità di bevande, naturalmente latte e suoi derivati, ma non soltanto: «in inverno fanno un'ottima bevanda di riso, miglio, frumento o miele, limpida come il vino; il vino lo importano da paesi lontani; in estate l'unica bevanda che interessa loro è il qumiz» $\gg^{10}$. La civiltà dei Mongoli, scopre Guglielmo a poco a poco, è una civiltà del bere: accanto alla porta della dimora dei signori è posto un otre di latte, da cui tutti si servono ${ }^{11}$, i rituali propiziatori comportano l'aspersione di bevande ${ }^{12}$, l'ambasciatore

7 Itiner. V 1.

8 «In sero dabant nobis carnes, scapulam arietis cum costis, et de brodio ad mensuram bibere. Quando habebamus de brodio carnium ad saturitatem, optime reficiebamur, et videbatur michi sanissimus potus et maxime nutriens. Feria sexta permanebam ieiunus usque ad noctem, nichil hauriens; tunc oportebat me cum tristitia et dolore comedere carnes» (Itiner. XXII 1).

9 «Tartari nesciunt eos capere nec curant de pisce, nisi sit ita magnus quod possint comedere carnes eius sicut carnes arietinas» (Itiner. XIII 10); nello stesso capitolo si parla dei pescatori russi (Ruteni) del Don. In realtà quando si troverà alla corte mongola a Guglielmo verranno propinati anche «pisces magni, qui dicuntur carpes, sine sale, sine pane» (Itiner. XXIX 22), che assaggerà soltanto («de quibus pauca comedi»).

10 «Faciunt in hyeme optimum potum, de risio, de millio, de tritico, de melle, clarum sicut vinum; et defertur eis vinum a remotis partibus. In estate non curant nisi de comos» (Itiner. II 9).

11 Itiner. II 9: «Bancus cum utre lactis vel cum alio potu stat semper infra domum ad introitum porte».

$12 \ll$ Et cum convenerunt ad potandum primo aspergunt de potu illi ymagini 
non può parlare a corte se non dopo aver bevuto ${ }^{13}$. Tutto è preceduto da un brindisi.

I Mongoli, che abitano nelle yurte, hanno un unico palazzo, che è quello che il sovrano usa per rappresentanza, nella città di Caracorum, senza per altro abitarvi. In questo palazzo, l'oggetto più prezioso è una fontana che eroga quattro bevande: il qumiz, naturalmente, nella sua varietà più pregiata (il qumiz nero), e poi birra di riso, idromele, vino. Guglielmo rimane incantato da questa fontana, opera di un artigiano francese che i Mongoli avevano preso prigioniero durante le loro scorrerie nell'Europa orientale, e la descrive con dovizia di particolari ${ }^{14}$; essa viene messa in funzione in occasione delle feste, nelle quali, naturalmente, la parte più importante è riservata al bere ${ }^{15}$.

Guglielmo non si limita a descrivere i cibi dei Mongoli, ma parla anche ampiamente dei rituali alimentari:

Con la carne di un solo montone danno da mangiare a cinquanta o cento uomini. La riducono in pezzi in un recipiente con sale e acqua - non usano altre salse -, poi con la punta di un coltello o una forchetta fatta apposta, come quelle che noi usiamo per mangiare le pere o le mele cotte nel vino, danno a ciascuno dei presenti uno o due bocconi, secondo il numero dei commensali. Il signore, davanti al quale è posta la carne del montone, prende per primo il pezzo che più gli aggrada. Se intende dare a qualcuno una parte speciale, la regola vuole che questi la prenda e la mangi lui solo, e non gli è permesso darne a nessuno: se non riesce a mangiare tutta la porzione, può portarla via o darla a un suo servo, se è presente, perché gliela tenga da parte, oppure la può mettere nel suo captargac, una bisaccia quadrata che usano per riporre cose del genere (vi mettono perfino le ossa, quando non hanno tempo

que est super capud domini, postea aliis ymaginibus per ordinem. Postea exit minister domum cum cipho et potu et spargit ter ad meridiem, qualibet vice flectendo genu, et hoc ad reverentiam ignis; postea ad orientem, et hoc ad reverentiam aeris; postea ad occidentem ad reverentiam aque; ad aquilonem proiciunt pro mortuis. Quando tenet dominus ciphum in manu et debet bibere, tunc primo ${ }^{12}$ infundit terre partem suam. Si bibat sedens super equum, infundit antequam bibat super collum equi. Postquam ergo minister sic sparserit ad quatuor latera mundi revertitur in domum, et sunt parati duo famuli cum duobus ciphis et totidem patenis ut deferant potum domino et uxori sedenti iuxta eum sursum in lecto» (Itiner. II 8).

13 Itiner. XXVIII 15.

14 Itiner. XXX 2-3.

15 Anche altri divertimenti ruotano intorno al rituale del bere; in Itiner. II 10 Guglielmo descrive una curiosa cerimonia con cui si spinge a bere chi è riottoso a farlo. 
di spolparle bene, per poterlo fare in un altro momento, in modo che nulla del cibo vada perduto). ${ }^{16}$

Questo modo di raccontare dimostra che Guglielmo è consapevole di misurare col cibo la distanza fra due civiltà. Attraverso il cibo si imparano delle regole, e queste regole sono specchio di valori. In questo caso ci viene presentato un rituale di potere, secondo il quale il capo prende il pezzo migliore, poi sceglie altre parti pregiate per gli uomini che vuole premiare. Questi bocconi speciali non possono essere dati ad altri, né gettati, ma chi li ha ricevuti, se non riesce a finirli, li conserva nella sua bisaccia, e continua a mangiarli finché non li ha finiti; un rituale nel quale il risparmio e la valorizzazione del cibo, una risorsa rara, si intreccia con la magia e il segreto che promana dalla concessione del capo. Tutti gli altri mangiano insieme dal pentolone, in quello che potrebbe essere una forma di egualitarismo popolare. Se egualitarismo era, Guglielmo lo percepisce solo in parte, e sembra rimanere un po' scandalizzato per la promiscuità; così come - forse a maggior diritto, almeno secondo un'ottica moderna - si scandalizza per la pessima igiene delle attrezzature da tavola:

Non lavano mai le stoviglie: anzi, dopo che hanno cotto la carne, sciacquano con il brodo bollente della pentola il recipiente dove devono versarla, e poi rimettono il brodo nella pentola. ${ }^{17}$

Che il cibo abbia forti valori simbolici - e che il "cibo degli altri"

16 «De carne unius arietis dant comedere L hominibus vel C. Scindunt enim minutatim in scutella cum sale et aqua, aliam enim salsam non faciunt, et tunc cum puncta cutelli vel fuscinula quas proprias faciunt ad hoc, cum quibus solemus comedere pira et poma cocta in vino, porrigunt cuilibet circumstantium bucellam unam vel duas, secundum multitudinem comedentium. Dominus, ante quem ponitur caro arietis, in primo ipse accipiet quod placet ei; et etiam si dat alicui partem specialem, oportet quod accipiens comedat eam solus, et nemini licet ei dare, sed si non potest totum comedere, asportet secum vel det garcioni suo, si est presens, qui custodiat ei: sin autem, recondit in captargac suo, hoc est bursa quadrata, quam portant ad recondendum omnia talia, in qua etiam ossa recondunt, quando non habent spatium bene rodendi ea, ut postea rodant ne pereat aliquid de cibo» (Itiner. III 2).

17 «Numquam etiam lavant scutellas, immo, carne cocta, alveum in quo debent ponere eam lavant brodio bulliente de caldaria, et postea refundunt in caldariam» (Itiner. VII 1 bis). 
li abbia ancora di più, data la sua inusualità - lo si sa del resto da sempre, da molto prima che l'antropologia ce ne abbia data definizione. La stessa guida che offre per prima il qumiz a Guglielmo - un mongolo privo di cultura, agli occhi del nostro viaggiatore un vero selvaggio - ne è consapevole: la sua offerta è fatta appena oltre il confine, in segno di accoglienza e di condivisione. Ma, per le stesse ragioni e gli stessi meccanismi, il cibo può essere talvolta, al contrario, non un positivo strumento di crescita e di conoscenza, ma il modo di verificare la profondità di una divisione, e diventare il simbolo di un'irrimediabile separatezza.

Il secondo caso che presentiamo è quello di un altro viaggiatore che ci ha lasciato il suo racconto: Liutprando, diacono di Pavia e poi vescovo di Cremona nel $\mathrm{X}$ secolo, due volte ambasciatore a Costantinopoli, la prima nel 949-50 per Berengario, marchese d'Ivrea e ben presto re d'Italia come Berengario II, la seconda nel 968 per l'imperatore Ottone I di Sassonia ${ }^{18}$. La destinazione, a confronto con quella di Guglielmo di Rubruk, può apparire deludente: il viaggio a Costantinopoli è meno esotico di quello in Mongolia, e certo l'esperienza di Bisanzio, per quanto vi si vivesse in modo diverso dalle città dell'Italia o dai castelli della Germania, era meno straniante di quella di un ambasciatore latino nell'Asia centrale. Ma Liutprando, a differenza di Guglielmo, vive in un'epoca in cui i viaggi erano molto più rari e non meno avventurosi, e l'impressione che desta un'esperienza, come si sa, è inversamente proporzionale alla frequenza con cui si può realizzare. Anche Liutprando, come Guglielmo, racconta il suo viaggio - anzi, come vedremo, i suoi viaggi - come protagonista, facendocene sentire l'emozione; e nei suoi racconti il cibo ha, anche stavolta, una parte di primo piano.

Le affinità, però, terminano qui. A differenza di Guglielmo, che secoli dopo narrerà il suo viaggio in una dimensione reale - e in questo è la bellezza del suo scritto -, Liutprando ci racconta un viaggio interpretato, e in questo sta la sua suggestività. Intendiamoci: le due ambascerie di cui egli fu protagonista sono fatti storici, e anche le vicende personali che riferisce saranno, fatta salva qualche inevitabile distorsione o esagerazione, realmente avvenute. Ma lo scopo di Liutprando non

$18 \mathrm{Su}$ Liutprando e le sue vicende rimandiamo all'ottima introduzione di Bougard 2015. Le opere di Liutprando verranno citate dall'edizione da noi curata (Chiesa 1998); la traduzione dei brani dell'Antapodosis è tratta da Chiesa 2015. 
è quello di riferire un'esperienza: i suoi racconti hanno una finalità pubblicistica evidente, nel primo caso a esaltazione dell'interlocutore bizantino, nel secondo a sua denigrazione. Nella prima ambasceria, quella del 949-50, egli è mandato da un padrone che non sopporta (Berengario) e incontra un imperatore bizantino di cui dice meraviglie (Costantino VII Porfirogenito); nella seconda ambasceria, del 968, egli è mandato dal sovrano a cui deve tutto (Ottone) e incontra un imperatore bizantino che rappresenta come un mostro (Niceforo II Foca). Nel secondo caso il racconto - cui è dedicato un intero opuscolo, la Relatio de relatione Constantinopolitana, o più semplicemente Legatio - è scopertamente finalizzato a giustificare il proprio fallimento: gli obiettivi diplomatici per i quali l'aveva inviato Ottone non sono stati raggiunti, ma la colpa, dice Liutprando, è tutta della controparte bizantina. Nel primo caso, la ragione della narrazione non è altrettanto chiara, anche perché l'opera in cui si legge, l'Antapodosis - una cronaca incompiuta di vicende varie che occupano una cinquantina d'anni - si interrompe bruscamente proprio con quel racconto. L'intento di Liutprando, nel sottolineare la grandezza di Costantino VII, potrebbe essere quello di censurare la meschinità di Berengario, descritto come un piccolo nobile tirchio; ma di recente è stata avanzata anche la suggestiva ipotesi che egli abbia introdotto quella storia 'a posteriori', dopo la fallita ambasceria del 968, in un'opera che aveva scritto in precedenza, per rimarcare ancora di più, con un confronto impietoso, la decadenza e lo squallore della corte di Niceforo, a confronto con la magnificenza di quella di Costantino, neanche vent'anni prima ${ }^{19}$.

In questo gioco di esaltazione la prima volta, di denigrazione la seconda, il cibo riveste una funzione iconografica decisiva ${ }^{20}$. Arrivato a Costantinopoli nel primo viaggio, Liutprando viene invitato dall'imperatore a un sontuoso banchetto che viene descritto così:

Poco a nord dell'ippodromo si trova un palazzo di straordinaria altezza e bellezza, che si chiama Dekanneacubita. Il nome non è casuale, ma deriva da ragioni evidenti. Deka in greco significa "dieci", ennea significa "nove", cubita deriva da cubare e significa "sdraiato" o "piegato"; il nome del palazzo dipende dal fatto che nel giorno della Natività di nostro Signore Gesù Cristo vi vengono

19 Così Arnaldi in Chiesa 2015, XXXVII-XXXIX.

20 Un'esauriente analisi dei cibi e delle abitudini alimentari bizantine come presentate da Liutprando è quella di Weber 1980. 
poste diciannove mense, alle quali l'imperatore e gli altri commensali mangiano non seduti, come negli altri giorni, ma sdraiati, e non vengono usati recipienti d'argento, ma soltanto d'oro. Al termine del pranzo vengono portati dei frutti in tre vasi d'oro, così pesanti da non poter essere recati a mano da servi, tanto che arrivano su carrelli ricoperti di porpora. Due di tali vasi vengono poi sistemati sulla mensa in questo modo: attraverso dei fori nel soffitto sono calate tre funi ricoperte di pelle dorata, con degli anelli d'oro all'estremità; questi anelli sono fatti passare nelle anse che sporgono dai vasi, ed essi sono sollevati e quindi deposti sopra la mensa, con l'impiego di una carrucola girevole che si trova sopra il soffitto, da quattro e più uomini che tirano la fune dal basso ${ }^{21}$.

Tutto, qui, è straordinario (Liutprando usa il superlativo thaumastotaton, secondo il suo vezzo di impiegare talvolta espressioni greche quia sonorius est ${ }^{22}$, cioè per creare una sottolineatura stilistica). La grandezza architettonica del palazzo; la sua bellezza; le mense cui i commensali mangiano sdraiati, alla maniera degli antichi romani e a differenza degli europei dell'epoca; le stoviglie d'oro; i vasi pieni di frutta, così pesanti da non poter essere portati a mano; i meccanismi di scena, la carrucola sopra il soffitto, le corde dorate, la deposizione dei vassoi sulla mensa. L'ambientazione eccezionale porta con sé un'esotismo fiabesco, in cui si misura la distanza dai possibili elementi di confronto: davanti alla magnificenza del banchetto di Costantino, Berengario, il suo mandante (e nemico) occidentale, che per avarizia ha inviato l'ambasciatore a mani vuote, rimpicciolisce a dismisura; e Niceforo Foca - se fosse lui l'obiettivo polemico a distanza - con la grottesca sciatteria dei suoi squallidi pseudo-banchetti, precipita in un abisso di decadenza.

21 «Est domus iuxta yppodromum aquilonem versus mirae altitudinis seu pulchritudinis, quae Decanneacubita vocatur. Quod nomen non ab re, sed ex apparentibus causis sortita est: deca enim Grece, Latine X, ennea IX, cubita autem a cubando inclinata vel curvata possumus dicere; hoc autem ideo, quoniam quidem $\mathrm{X}$ et IX mensae in ea, quae secundum carnem est, Domini nostri Iesu Christi nativitate apponuntur, in quibus imperator pariter et convivae non sedendo, ut caeteris diebus, sed recumbendo epulantur; quibus in diebus non argenteis, sed aureis tantum vasis ministratur. Post cibum autem aureis vasis tribus sunt poma delata, quae ob inmensum pondus non hominum manibus, sed purpura tectis vehiculis sunt allata. Apponuntur autem duo hoc in mensam modo. Per foramina laquearis tres sunt funes pellibus deauratis tecti cum anulis depositi aureis, qui ansis, quae in scutulis prominent, positi, adiuvantibus inferius quattuor aut eo amplius hominibus, per vertibile, quod supra laquear est, ergalium in mensam subvehuntur eodemque modo deponuntur» (Antap. VI 8).

22 Antap. II 34. 
Perché è appunto questa la ben diversa atmosfera e la ben diversa accoglienza che Liutprando troverà - almeno a suo dire - diciannove anni dopo, alla corte di Niceforo Foca; e ben diversi sono com'è ovvio anche i cibi. In verità, non si può dire che Niceforo abbia lasciato il nostro ambasciatore senza mangiare: lo invitava anzi a pranzo abbastanza spesso, ma l'ambiente era questo:

Quel giorno ... [l'imperatore] ordinò che fossi ospite alla sua tavola. Non mi ritenne però degno di essere preposto a qualcuno dei suoi cortigiani, e venni messo a sedere a quindici posti di distanza da lui, senza neppure la tovaglia; dei miei compagni nessuno poté non dico sedere a tavola, ma neppure vedere la casa in cui ero ospite. Durante questa cena - pessima e volgare come una gozzoviglia fra ubriachi, intrisa d'olio e innaffiata da un'altra abominevole broda di pesce - egli continuò a farmi domande sulla vostra potenza, i vostri regni e i vostri uomini. ${ }^{23}$

Altro che vasi calati in tavola con corde d'oro! Prima ancora del cibo - condito con la salsa di pesce, discendente del celebre garum che i Bizantini avevano ereditato dagli antichi Romani insieme al titolo imperiale - a Liutprando dà fastidio l'apparato cerimoniale: la tavola senza tovaglia, la posizione defilata in cui l'hanno relegato, l'atteggiamento inquisitorio del padrone di casa. Ancor più del missionario Guglielmo, l'ambasciatore Liutprando è consapevole dei significati simbolici del cibo e della tavola, e a questi richiama l'imperatore Ottone e i membri della sua famiglia, cioè i destinatari dichiarati della Legatio, nel raccontare un successivo invito a pranzo di Niceforo:

Dopo stucchevoli litanie e dopo la celebrazione dei sacri riti, veniamo invitati a pranzo; e a tavola - la solita tavola lunga e strettissima - Niceforo assegna un posto migliore del mio, sul lato più vicino della mensa, all'ambasciatore dei Bulgari, un uomo rasato alla maniera degli Ungari, con una catena al collo che pareva uno schiavo, a quel che sospetto nemmeno battezzato; di sicuro lo fece per recare oltraggio a voi, miei signori imperatori. ${ }^{24}$

23 «Hac eadem die convivam me sibi esse iussit. Non ratus autem me dignum esse cuipiam suorum praeponi procerum, quintus decimus ab eo absque gausape sedi; meorum nemo comitum, non dico solum mensae non assedit, sed neque domum in qua conviva eram, vidit. Qua in coena, turpi satis et obscena ebriorum more, oleo delibuta alioque quodam deterrimo piscium liquore aspersa, multa super potentia vestra, multa super regnis et militibus me rogavit» (Legatio XI).

24 «Cumque post naeniarum garrulitatem et missarum celebrationem ad mensam 
E di fronte a quello che ritiene un oltraggio - l'ambasciatore dei Bulgari messo davanti a quello dell'imperatore d'Occidente! Liutprando si alza e vuole andarsene, ma lo relegano in cucina a mangiare coi servi:

Ma quel santo imperatore temperò il mio dolore con un grande dono, mandandomi, dei suoi cibi così raffinati, un pingue capretto di cui si era servito anche lui, sontuosamente farcito di agli, cipolle e porri, intriso di broda di pesce. Avrei voluto che questa pietanza squisita fosse portata alla vostra tavola: se non credete che le leccornie di quel santo imperatore siano eccellenti, ve ne sareste potuti sincerare di persona. ${ }^{25}$

L'ironia sul cibo è violenta. La capra in Occidente era considerato un cibo da contadini, non certo da sovrani, e da poveri erano anche aglio, cipolle e porri; e non si deve credere - dice Liutprando - che quello mandato a lui fosse un piatto secondario, riservato a ospiti di basso rango, perché lo stesso Niceforo se ne era servito. Un imperatore rozzo, che si fa servire cibi dozzinali: impossibile svolgere con successo missioni diplomatiche presso gente del genere.

Il pessimo cibo diventa perciò il simbolo di una pessima società e di un pessimo governo: e Liutprando vi insisterà ancora, quando verrà invitato a un'ennesima cena «dal buon profumo di aglio e cipolla, bisunta d'olio e salsa di pesce ..., alla solita tavola lunga e strettissima, coperta da tovagliette nel senso della larghezza, praticamente scoperta in quello della lunghezza» ${ }^{26}$. Il cibo diventa qui segno identificativo di una civiltà decaduta, e per contrasto proietta più in alto la sana superiorità dell'Occidente, rappresentata da Ottone e dalla sua famiglia: «O

invitaremur, in citeriori mensae margine, quae erat sine latitudine longa, Bulgarorum nuntium, Ungarico more tonsum, aenea catena cinctum et - ut mens mihi suggerit catechumenum, mihi praeponit, ad vestram plane, domini mei augusti, contumeliam» (Legatio XIX).

25 «Sed lenivit dolorem meum imperator sanctus munere magno, mittens mihi ex delicatissimis cibis suis haedum pinguem ex quo ipse comederat, allio, cepe, porris laute suffarcinatum, garo delibutum, quem vestrae tunc mensae inesse optavi, ut, qui delicias sancti imperatoris faustas esse non creditis, saltem his perspectis crederetis» (Legatio XX).

26 «Me ... ad cenam allio et cepa bene olentem, oleo et garo sordidam, venire praecepit ... ad mensam sine latitudine longam, pallingi latitudine tectam, longitudine seminudam (Legatio XXXII-XXXIII)». 
voi miei signori imperatori augusti, voi sempre raffinati, quanto mi siete sembrati più raffinati da quel giorno! $\gg^{27}$.

Il più celebre fra i viaggiatori medievali è certo Marco Polo. Il libro che racconta il suo viaggio, il Devisement $d u$ mond, scritto dalla penna esperta del romanziere Rustichello da Pisa e al pubblico italiano più noto come Milione, è anche un grande repertorio sull'alimentazione dei popoli asiatici ${ }^{28}$. Marco vede il mondo con gli occhi del mercante, e spesso la sua attenzione agli alimenti va letta in questa particolare prospettiva. Per molte delle località che attraversa egli ci dice qual è il prodotto caratteristico, quello che si trova in abbondanza e si vende a buon prezzo, quello che un mercante occidentale potrebbe utilmente acquistare sul posto. Il suo libro prefigura così potenziali linee di commercio, che hanno per oggetto ora la carne, ora il pesce, ora la cacciagione, ora le più svariate farine, ora la frutta secca, e poi datteri, meloni, pistacchi, chiodi di garofano, corbezzoli, e via dicendo, fino al 'frutto del paradiso', quello che noi chiamiamo banana ${ }^{29}$. Il Milione, anche a dispetto del tono brillante e romanzesco che si deve a Rustichello, un tono che toglie un po' di quella genuinità di sentimento che avvertiamo nel racconto di viaggio

27 Legatio III.

28 Dell'opera citeremo la cosiddetta Versione toscana (da Bertolucci Pizzorusso 1975); la Versione ramusiana (dalla ristampa curata da Milanesi 1980); e la versione latina del codice Zelada (da Barbieri 1998).

29 I primi europei che ebbero conoscenza della banana lo chiamarono 'frutto del paradiso' perché essa, quando viene tagliata sul lato più corto, mostra all'interno $i$ semi disposti approssimativamente a forma di croce; per questa ragione venne da alcuni identificata con il frutto del peccato di Adamo ed Eva. Così ad esempio la banana, chiamata musa, è descritta da un altro viaggiatore trecentesco, Giovanni di Marignolli: «Musa autem magis videtur planta hortensis quam arbor. Est enim grossa arbor, sicut quercus, et tante teneritudinis, quod fortis homo posset eam digito perforare, et exit de ea aqua continue. Folia istius muse sunt pulcherrima, longa et lata valde, viriditatis smaragdine, ita quod de foliis illis faciunt tobalias, in uno prandio tantum; quando etiam primo nascuntur pueri, post lotionem conditos sale et aloes et rosis, involvunt eos sine fascia in foliis istis, et in arenam ponunt; folia illa sunt longitudinis secundum magis et minus bene decem ulnarum, et similitudinem nescimus ponere nisi enule campane. Fructum producit tantum in summitate, et in uno baculo faciunt bene trecentos fructus, et prius non valent ad comedendum; post applicantur in domo et sunt optimi odoris et melioris saporis, et sunt longi ad modum longorum digitorum manus, et per se stando maturantur. Et istud vidimus oculis nostris, quod ubicumque inciditur per transversum, in utraque parte incisure videtur ymago hominis crucifixi, quasi si homo cum acu sculpsisset; et de istis foliis ficus Adam et Eva fecerunt sibi perizomata ad cooperiendum turpitudinem suam». (da Malfatto). 
di Guglielmo di Rubruk, è incredibilmente ricco e vario, perché moltissimi sono i luoghi che Marco ha attraversato - o dice di aver attraversato - e di cui ci parla; e il cibo tipico di ciascuna località ne diventa anche una marca identificativa. Il Milione è insomma il primo libro del medioevo a tracciare una sorta di geografia alimentare: l'esperienza è qui di larga scala, e può raggiungere una dimensione enciclopedica.

Marco Polo ci racconta ad esempio che della provincia di Tenduc, nel nord della Cina, sono tipiche le pernici, e ne è ghiotto il gran khan, che in estate soggiorna volentieri in questa regione perché può mangiarne in quantità, e che in inverno da lì se le fa portare $e^{30}$; che nell'isola di Madagascar non si usa altra carne che quella di cammello, secondo gli abitanti del posto la più sana e la migliore che vi sia al mondo ${ }^{31}$; che nella zona dei monti Urali si fabbrica una quantità di birra $^{32}$; che nella maggior parte della Cina si beve ottimo vino di riso ${ }^{33}$; che nel Caragia (Yun-nan) si mangia carne cruda ${ }^{34}$; e via dicendo. $\mathrm{Ci}$ riferisce talvolta anche vere e proprie ricette, come per esempio questa,

$30 \ll$ E presso a questa città è una valle, nella quale è grandissima abondanza di pernici e quaglie, e pel nutrimento delle qual sempre il gran Can fa seminar l'estate sopra quelle coste miglio e panizzo e altre semenze che tali uccelli appetiscono, comandando che niente si raccolga, acciò abondevolmente si possano nudrire... E ha fatto fare il gran Can molte casette dove stanno la notte, e quando 'l vien a questa contrada ha di questi uccelli abondantemente, e l'inverno, quando sono ben grasse (perché ivi pel gran freddo non sta a quel tempo) ovunque egli si sia, se ne fa portare carghi i camelli» (Versione ramusiana, I 54).

31 «E sapiate che in questa isola non si mangia altra carne che di camelli, e mangiavisene tanti che non si potrebbe credere; e dicono che questa carne di camelli è la più sana carne e la migliore che-ssia al mondo» (Versione toscana, CLXXXVI 6).

32 «Ipsi quidem vinum conficiunt de melle et panigio perfectissimum, quod cerbesia appelatur; et ad istam cerbesiam maximas potationes faciunt» (versione del codice Zelada, CLXV 36). Il brano prosegue descrivendo con dovizia di particolare quelli che i redattore del testo chiama straviza, ossia gli sfrenati festini collegati a queste bevute.

33 «Ancora sappiate che la magiore parte del Catai beono uno cotale vino com'io vi conterò. Egli fanno una pogione di riso e co molte altre buone spezie, e cóncialla in tale maniera ch'egli è meglio da bere che nullo altro vino. Egli è chiaro e bello, e inebria più tosto ch'altro vino, perciò ch'è molto caldo» (Versione toscana, C 1-3).

34 «Egli mangiano la carne cruda $<$ e $>$ ogne carne. E' poveri vanno a la beccheria, e quando s'apre il castrone o bue, sì li cavan le budella di corpo, e mettole ne la salsa de l'aglio e màngialle; e così fanno d'ogne carne. E li gentili uomini la mangian cruda, ma sì la fanno minuzzare molto minuto; poscia la mettono ne la salsa e màngialla e con buone spezia; e màngialla così come noi la cotta» (Versione toscana, CXVII 16-18). 
tipica della regione di Fransur o Fanfur (identificata come una zona delle isole della Sonda):

In questa provincia cavano farina d'arbori, perché hanno una sorte d'arbori grossi e longhi, alli quali levatali la prima scorza, ch'è sottile, si truova poi il suo legno grosso intorn'intorno per tre dita, e tutta la midolla di dentro è farina come quella del carvolo: e sono quegli arbori grossi come potrian abbracciar due uomini. E mettesi questa farina in mastelli pieni d'acqua, e menasi con un bastone dentro all'acqua: allora la semola e l'altre immondizie vengono di sopra, e la pura farina va al fondo. Fatto questo si getta via l'acqua, e la farina purgata e mondata che rimane s'adopra, e si fanno di quella lasagne e diverse vivande di pasta, delle qual ne ha mangiato più volte il detto messer Marco, e ne portò seco alcune a Venezia, qual è come il pane d'orzo e di quel sapore. ${ }^{35}$

Marco Polo porta dunque a Venezia un souvenir, delle gallette possiamo pensare - fatte con la farina di quell'albero del pane: è il cibo degli altri, stavolta, a venire da noi. Anche se nella fattispecie si tratta di un evento occasionale, e ciò che è importato è un semplice campione, era in questo modo che si poteva avviare un nuovo commercio: se il prodotto piaceva, se 'aveva mercato' fra i ricchi occidentali, esso poteva diventare un esotica ricercatezza, al pari delle spezie e delle stoffe pregiate. Non bisogna dimenticare che anche in questo modo il 'cibo degli altri', creando intersezioni e contaminazioni, e producendo ricchezza, contribuì allo sviluppo culturale e civile dell'intera Europa.

\section{BIBLIOGRAFIA}

BARBIERI 1998 - A. Barbieri, Marco Polo. Milione. Redazione latina del manoscritto Z, Parma, Guanda, 1998.

Bertolucci Pizzorusso 1975 - V. Bertolucci Pizzorusso, Marco Polo, Milione. Versione toscana del Trecento, indice ragionato a cura di G.R. Cardona, Milano, Adelphi, 1975.

BougARD 2015 - F. Bougard, Liudprand de Crémone. Euvres, Paris, CNRS éditions, 2015.

CHIESA 1998 - P. Chiesa, Liudprandi Cremonensis Opera omnia, Corpus Christianorum. Continuatio mediaevalis 156, Turnhout, Brepols, 1998.

ChIESA 2010 - P. Chiesa, Guglielmo di Rubruk. Viaggio in Mongolia, Roma-Milano, Mondadori, 2010.

35 Versione ramusiana, III 16. 
Chiesa 2015 - P. Chiesa, Liutprando. Antapodosis, Roma-Milano, Mondadori, 2015.

Malfatto - I. Malfatto, Le digressioni sull'Oriente nel «Chronicon Bohemorum» di Giovanne de' Marignolli, in http://ecodicibus.sismelfirenze.it/index.php/iohannes-de-marignollis-chronicon-bohemorum-excerpta-de-rebus-orientalibus; dc

MilaneSI 1980 - M. Milanesi, G.B. Ramusio. Navigazioni e viaggi, III, Torino, Einaudi, 1980, 79-297.

WeBER 1980 - Th. Weber, Essen und Trinken im Konstantinopel des 10. Jabrhunderts, nach den Berichten Liutprands von Cremona, in J. Koder - Th. Weber (hrsg.), Liutprand von Cremona in Konstantinopel. Untersuchungen zum griechischen Sprachschatz und zu realienkundlichen Aussagen in seinen Werken, Wien, Verlag der Österreichischen Akademie der Wissenschaften, 1980, 71-99 